\title{
A Failure-rate-reduction Periodic Preventive Maintenance Model with Delayed Initial Time in a Finite Time Period
}

\author{
Chun-Yuan Cheng \\ Department of Industrial Engineering and Management, \\ Chaoyang University of Technology, Taiwan, R.O.C. \\ XuFeng Zhao \\ Department of Mechanical and Industrial Engineering \\ Qatar University, Qatar \\ Mingchih Chen \\ Graduate Institute of Business Administration (Ph.D. Program), \\ Fu Jen Catholic University, Taiwan, R.O.C. \\ Te-Hsiu Sun* \\ Department of Industrial Engineering and Management, \\ Chaoyang University of Technology, Taiwan, R.O.C.
}

\begin{abstract}
A new machine will not fail easily in the early stage of its useful life. This phenomenon is consistent with the fact that the optimal sequential preventive maintenance (PM) policy which has longer PM intervals in its earlier stage of lifetime. In this paper, we propose a failure-ratereduction periodic PM model with delayed initial time in a finite time span. Then, the optimal periodic PM policy is developed by minimizing the expected total maintenance cost, which can have smaller expected total maintenance cost than the optimal policy of the original failure-ratereduction periodic PM model. The algorithm of finding the optimal PM policy for the proposed PM model is developed. Finally, examples are illustrated to verify the optimal policies of the proposed new PM model.
\end{abstract}

Keywords: Periodic preventive maintenance, Failure rate reduction, finite time span.

*Corresponding author.

E-mail address: thsun@cyut.edu.tw. 


\section{Introduction}

For a deteriorating and repairable system or equipment, the preventive maintenance (PM) can decelerate the aging speed or reduce the failure rate and then restore the system to a younger state (Pham and Wang [6], Nakagawa [5]). In real world, a system’s useful life is normally finite. When an aged system is replaced by a new one, the new system may not have exactly the same conditions (such as characteristics, investment cost, and maintenance expenses) as the system of the previous replacement cycle. For example, a new personal computer will not have exactly the same type of CPU as the replaced old computer since the CPU of the replaced computer may be phased out. It seems that the PM model found in literature (e.g., Nakagawa [5] and Jayabalan and Chaudhuri [3]), which are under the assumption of infinite time span, might not be suitable for the above case.

There is only some literature relating to the PM models of finite time span. The PM models for the leasing equipments are the most common examples of the finite-time-span case. These models are usually defined with a periodic PM interval. Pongpech and Murthy [7] developed a periodic PM model with failure rate reduction for the leasing equipments in a finite time period. Cheng et al. [1] and Cheng and Liu [2] discussed the cases of fully-periodic and partiallyperiodic PM policies using the algorithm proposed by Pongpech and Murthy [7].

On the other aspect, a new system or machine will not fail easily in the early stage of its useful life. This phenomenon reflects the theoretical fact that the optimal policy of a sequential PM has longer PM intervals in the earlier stage of its useful lifetime (Nakagawa [5]). Thus, for a finite time span issue, in order to obtain a better PM policy, we consider the idea of delaying the initial time of a periodic PM program where the periodic PM activities can be arranged more easily and conveniently than the sequential or non-periodic PM activities. The purpose of this research is to apply the above idea to develop a periodic PM model whose optimal PM policy has 
smaller expected total maintenance cost $(T C)$ than the optimal PM policy of the comparative PM model.

In this paper, the proposed PM model is compared to the PM model developed by Pongpech and Murthy [7] and Cheng and Liu [2] which is noted as "the original PM model" and is illustrated in Figure 1. In the original PM model, it can be found that the searching range of the PM interval $(T)$ for any given number of PM $(N)$ is constrained in the range of [ $\left.T_{\min }, T_{\max }\right)$ where $T_{\min }=L /(N+1)$ and $T_{\max }=L / N$. Let $\pi$ be the time interval between $L$ and the time of the $N^{\text {th }} \mathrm{PM}$, i.e., $\pi=L-N T$. It turns out that $\pi \leq T$.

Next, the concept of the proposed periodic PM model with delayed initial time for the case of failure rate reduction is illustrated in Figure 2 which is noted as "the delayed PM model". It can be seen that the initial time of the PM program is not at time zero. It is assumed that the first PM is performed at time $\tau$ where $\tau \geq T$, i.e., the initial time of the PM program is at time $\tau-T$. We hypothesize that the delayed PM model can provide a better optimal solution than the original PM model.

The optimal solution of the proposed PM model is determined by minimizing the expected total maintenance cost (TC) of the finite time span. The theoretical proof of optimization solution is provided. The algorithm of finding the optimal solution is also developed. Examples of Weibull failure case are constructed for the proposed PM model to examine the hypothesis and to analyze the sensitivity of optimal solution.

\section{The Delayed PM Model with Failure Rate Reduction}

For the delayed PM model, the improvement factor introduced by Malik [4] is applied to measure the PM restoration effect. Since a deteriorating system or equipment with a useful finite life time is considered in this research, the minimal repair cost and the PM cost over the finite life time are the necessary elements of the cost function in the delayed PM model. 


\subsection{Nomenclature}

$L \quad$ The useful life time (finite time span) for the system or equipment.

$T \quad$ The time interval of each periodic PM.

$N \quad$ The number of PM cycles in the finite life time span $(L)$.

$\eta \quad$ The improvement factor for measuring the effect of PM restoration where $0 \leq \eta \leq 1$.

$\tau \quad$ The time of the first PM where $\tau \geq 0$.

$\lambda(t) \quad$ The original hazard rate function at time $t$ (before the first PM).

$\lambda_{i}(t) \quad$ The hazard rate function at time $t$ of the $i^{t h} \mathrm{PM}$ cycle and $\lambda_{0}(t)=\lambda(t)$.

$\Lambda_{i}(t)=\Lambda_{i}(t)=\int_{0}^{t} \lambda_{i}(u) d u$ which is the expected number of failure at time $t$ of the $i^{t h} \mathrm{PM}$ cycle and $\Lambda_{0}(t)=\Lambda(t)=\int_{0}^{t} \lambda(u) d u$.

$d_{N}(\tau) \quad=\min _{1 \leq i \leq N}\left\{\frac{\lambda(\tau+(i-1) T)}{i}\right\}$ which is the upper limit of the quantity of the failure rate reduction for each PM

$\delta_{N}(\tau, \eta)$ The quantity of the restored failure rate after each $\mathrm{PM}$ when given $N$, which is affected the time of the first PM $(\tau)$ and the improvement factor $(\eta)$; the notation is simplified as $\delta_{N}(\tau)$ when $\eta=1$.

$C_{m r} \quad$ The minimal repair cost of each random failure.

$C_{p m}\left(i, \delta_{N}(\tau, \eta)\right)$ The PM Cost in the $i^{\text {th }}$ PM cycle, which is function of $i$ and $\delta_{N}(\tau, \eta)$.

$T C(N, \tau, \eta)$ The expected total maintenance cost over the finite life time interval $L$, the notation is simplified as $T C(N, \tau)$ when $\eta=1$.

\subsection{Assumptions}

The following are the assumptions for the delayed PM model. 
- The system is deteriorating over time with power law increasing failure rate (IFR) in which Weibull failure distribution is assumed in this paper, i.e.,

$$
\lambda(t)=\frac{\beta}{\theta}\left(\frac{t}{\theta}\right)^{\beta-1}
$$

where $\theta$ is the scale parameter and $\beta$ is the shape parameter with $\beta \geq 1$.

- The system is disposed at specified finite time $(L)$ without replacing a new one where the disposed system is assumed to have no salvage market value.

- The periodic PMs with constant time interval $(T)$ are performed over a finite time period where the PM can reduce the system's failure rate to a younger level and the PM interval is limited in the range $(0, L]$.

- The initial time of the PM program is assumed to be delayed and the first PM is performed at time $\tau$ where $\tau \geq 0$.

- The failure rate restored in each PM is assumed to have the same quantity $\left(\delta_{N}(\tau, \eta)\right)$ when given $N$, $\tau$, and $\eta$, and the failure rate function of the $i$ th $\mathrm{PM}\left(\lambda_{i}(t)\right)$ is defined as

$$
\lambda_{i}(t)=\lambda_{i-1}(t)-\delta_{N}(\tau, \eta)=\lambda(t)-i \delta_{N}(\tau, \eta)
$$

- Minimal repair is performed when a random failure occurs between each PM.

- The minimal repair cost $\left(C_{m r}\right)$ of each random failure is assumed to be constant.

- When given $N, \tau$, and $\eta$, each PM cost $\left(C_{p m}\right)$ is assumed to be variable, which is affected by the age (expressed by the number of PM performed) and the quantity of failure rate reduction, and is defined as the following equation.

$$
C_{p m}\left(i, \delta_{N}(\tau, \eta)\right)=a+b i+c \delta_{N}(\tau, \eta)
$$

where $a, b$, and $c$ are the coefficients of the PM cost function.

- The times to perform PMs and minimal repairs are negligible in the delayed PM model. 
- The minimal repair cost $\left(C_{m r}\right)$ and the PM cost $\left(C_{p m}\right)$ are assumed to include the following items: labor of repair and maintenance, material and parts, and loss of downtime in production and it is assumed that $C_{p m} \geq C_{m r}$ in this paper.

\subsection{Model Development}

In the delayed PM model, the number of PM $(N)$, the time of the first $\mathrm{PM}(\tau)$, and the improvement factor $(\eta)$ are the decision variables. Thus, the PM time interval $(T)$ can be obtained as $T=(L-\tau) / N$. Since the quantity of the restored failure rate is assumed to be equal for each PM, the upper limit of quantity of the failure rate restoration $\left(d_{N}(\tau)\right)$ for each PM should be the minimal value of all possible quantities restored in the entire PM cycles (see Fig. 2). The improvement factor $\eta$ represents the ratio (the effect) of the restoration limit $d_{N}(\tau)$. Therefore, the restored failure rate $\left(\delta_{N}(\tau, \eta)\right)$ in each PM can be determined by the following equation.

$$
\delta_{N}(\tau, \eta)=\eta d_{N}(\tau)=\eta \cdot \min _{1 \leq i \leq N}\left\{\frac{\lambda(\tau+(i-1) T)}{i}\right\}=\eta \cdot \min _{1 \leq i \leq N}\left\{\frac{\lambda\left(\tau+(i-1) \frac{L-\tau}{N}\right)}{i}\right\} .
$$

The expected total maintenance cost $(T C)$ of the delayed PM model is derived as follows.

$$
\begin{aligned}
T C(N, \tau, \eta) & =C_{m r}\left\{\int_{0}^{\tau} \lambda(t) d t+\sum_{i=1}^{N} \int_{\tau+(i-1) T}^{\tau+i T} \lambda_{i}(t) d t\right\}+\sum_{i=1}^{N} C_{p m}\left(i, \delta_{N}(\tau, \eta)\right) \\
& =C_{m r}\left\{\int_{0}^{\tau} \lambda(t) d t+\sum_{i=1}^{N} \int_{\tau+(i-1)\left(\frac{L-\tau}{N}\right)}^{\tau+i\left(\frac{L-\tau}{N}\right)}\left[\lambda(t)-i \delta_{N}(\tau, \eta)\right] d t\right\}+\sum_{i=1}^{N}\left[a+i b+c \delta_{N}(\tau, \eta)\right] \\
& =C_{m r} \int_{0}^{L} \lambda(t) d t+N\left[a+\frac{(N+1)}{2} b+c \delta_{N}(\tau, \eta)\right]-\frac{(N+1)}{2} \delta_{N}(\tau, \eta)(L-\tau) C_{m r}
\end{aligned}
$$

For Weibull failure case, the equation of $T C$ can be expressed in Eq. (6).

$$
T C(N, \tau, \eta)=C_{m r}\left(\frac{L}{\theta}\right)^{\beta}+N\left[a+\frac{(N+1)}{2} b+c \delta_{N}(\tau, \eta)\right]-\left(\frac{N+1}{2}\right) \delta_{N}(\tau, \eta)(L-\tau) C_{m r}
$$




\subsection{The Properties of the Model}

Theorem 1. If $F$ is an IFR distribution with strictly increasing and convex failure rate function $\lambda(t)$, for any given $\tau$ and $\eta$, the restored failure rate $\delta_{N}(\tau, \eta)$ shown in Eq.(4) is decreasing with $N$ increasing, i.e.,:

$$
\delta_{N}(\tau, \eta) \geq \delta_{N+1}(\tau, \eta)
$$

Proof. Since the failure rate function $\lambda(t)$ is a strictly increasing function, it can be obviously found that,

$$
\frac{\lambda\left(\tau+(i-1) \frac{(L-\tau)}{N}\right)}{i} \geq \frac{\lambda\left(\tau+(i-1) \frac{(L-\tau)}{N+1}\right)}{i}
$$

for the $i^{t h} P M$ where $i=1,2, \ldots, N$. It turns out that $\eta d_{N}(\tau) \geq \eta d_{N+1}(\tau)$ is affirmed and Eq.(7) is proved.

The optimal solution of the delayed PM model is to seek the optimal value of $\tau^{*}, \eta^{*}$ and $N^{*}$ which minimize the expected total maintenance cost $(T C(N, \eta, \tau))$ in Eq.(5) or (6). Consider that $\tau$ and $\eta$ are fixed. Then, we can obtain an optimal number $N^{*}$ by forming the inequalities

$$
T C(N+1, \eta, \tau) \geq T C(N, \eta, \tau) \text { and } T C(N, \eta, \tau)<T C(N-1, \eta, \tau)
$$

Theorem 2. There exists an $N^{*}<\infty$ which satisfies Eq. (8) for any given positive $\tau$ and $\eta$ and it is unique.

Proof. In Eq.(8), $T C(N+1, \eta, \tau) \geq T C(N, \eta, \tau)$ can be written as

$$
a+(N+1) b+\delta_{N+1}(\tau, \eta)\left[(N+1) c-\frac{(N+2)}{2}(L-\tau) C_{m r}\right]-\delta_{N}(\tau, \eta)\left[N c-\frac{(N+1)}{2}(L-\tau) C_{m r}\right] \geq 0 .
$$

According to Theorem 1, we know that $\delta_{N}(\tau, \eta) \geq \delta_{N+1}(\tau, \eta)$. Thus, the inequality of Eq.(9) remains valid if the term $\delta_{N+1}(\tau, \eta)$ is substituted by $\delta_{N}(\tau, \eta)$ and it becomes 


$$
a+(N+1) b-\delta_{N}(\tau, \eta)\left[\frac{1}{2}(L-\tau) C_{m r}-c\right] \geq 0 .
$$

Similarly, $T C(N, \eta, \tau)<T C(N-1, \eta, \tau)$ can be written as

$$
a+N b-\delta_{N}(\tau, \eta)\left[-N c+\frac{(N+1)}{2}(L-\tau) C_{m r}\right]+\delta_{N-1}(\tau, \eta)\left[-(N-1) c+\frac{N}{2}(L-\tau) C_{m r}\right]<0 .
$$

Based on Theorem 1, we know that $\delta_{N-1}(\tau, \eta) \geq \delta_{N}(\tau, \eta)$. Thus, the inequality of Eq.(11) remains valid if the term $\delta_{N}(\tau, \eta)$ is substituted by $\delta_{N-1}(\tau, \eta)$ and it becomes

$$
a+N b-\delta_{N-1}(\tau, \eta)\left[\frac{1}{2}(L-\tau) C_{m r}-c\right]<0 .
$$

Let

$$
G(N, \tau, \eta)=a+(N+1) b-\delta_{N}(\tau, \eta)\left[\frac{1}{2}(L-\tau) C_{m r}-c\right] .
$$

Therefore, Eq.(8) can be written as

$$
G(N, \tau, \eta) \geq 0 \text { and } G(N-1, \tau, \eta)<0
$$

Next, to prove that there exists a unique $N^{*}<\infty$ which satisfies Eq. (8) for any given $\tau$ and $\eta$, we need to verify that $G(N, \tau, \eta)-G(N-1, \tau, \eta)>0$ which is shown as follows.

$$
G(N, \tau, \eta)-G(N-1, \tau, \eta)=b+\left[\delta_{N-1}(\tau, \eta)-\delta_{N}(\tau, \eta)\right]\left(\frac{1}{2}(L-\tau) C_{m r}-c\right)
$$

Again, from Theorem 1, it can be found that $\left[\delta_{N-1}(\tau, \eta)-\delta_{N}(\tau, \eta)\right]>0$. Then, from Eq. (5), we can obtain that $\left(\frac{1}{2}(L-\tau) \mathrm{C}_{m r}-c\right)>0$ when $N^{*}>0$. Hence, the inequality $G(N, \tau, \eta)-G(N-1, \tau, \eta)>0$ is verified.

Theorem 3. For any given positive $\tau$ and $N>0$, the optimal value of improvement factor is $\eta^{*}$ $=1$. 
Proof. For given positive $\tau$ and $N>0, \eta^{*}$ can be obtained by taking partial derivative of Eq.(5) with respective to $\eta$ as follows.

$$
\frac{\partial T C(N, \tau, \eta)}{\partial \eta}=-d_{N}(\tau) N\left[\frac{N+1}{2 N}(L-\tau) C_{m r}-c\right]
$$

where $d_{N}(\tau)=\min _{1 \leq i \leq N}\left\{\lambda\left(\tau+(i-1) \frac{L-\tau}{N}\right) / i\right\}$ and $\left[\frac{N+1}{2 N}(L-\tau) C_{m r}-c\right]>\left[\frac{1}{2}(L-\tau) C_{m r}-c\right]>0$. It turns out that $\frac{\partial T C(N, \tau, \eta)}{\partial \eta}<0$ which implies that the larger the $\eta$ is, the smaller the $T C(N, \tau, \eta)$ will be. Since $\eta$ is in the range of $[0,1]$, the optimal value of $\eta$ is $\eta^{*}=1$.

According to Theorem 3, since $\eta^{*}=1$, it turns out that $\delta_{N}(\tau, \eta)=d_{N}(\tau)$. This means that the minimal value of all possible quantities restored in the entire PM cycles is the optimal quantity of failure rate restoration. Hence, $\delta_{N}(\tau, \eta)$ and $T C(N, \tau, \eta)$ can be simply noted as $\delta_{N}(\tau)$ and $T C(N, \tau)$, respectively, with the following equations.

$$
\begin{gathered}
\delta_{N}(\tau)=d_{N}(\tau)=\min _{1 \leq i \leq N}\left\{\frac{\lambda(\tau+(i-1) T)}{i}\right\}=\min _{1 \leq i \leq N}\left\{\frac{\lambda\left(\tau+(i-1) \frac{L-\tau}{N}\right)}{i}\right\} . \\
T C(N, \tau)=C_{m r} \int_{0}^{L} \lambda(t) d t+N\left[a+\frac{(N+1)}{2} b+c \delta_{N}(\tau)\right]-\frac{(N+1)}{2} \delta_{N}(\tau)(L-\tau) C_{m r}
\end{gathered}
$$

Theorem 4. For any given $N>0$, there exists a unique $\tau^{*}<L$ which satisfies

$$
\frac{\partial T C(N, \tau, \eta)}{\partial \tau}=0
$$

Proof. Applying Theorem 3 (i.e., $\eta^{*}=1$ ) to Eq.(5) which becomes Eq. (16) and taking partial derivative of Eq.(16) with respective to $\tau$, we can obtain

$$
\frac{\partial T C(N, \tau)}{\partial \tau}=\delta_{N}^{\prime}(\tau)\left[N c-\frac{N+1}{2}(L-\tau) C_{m r}\right]+\frac{N+1}{2} C_{m r} \delta_{N}(\tau)=0
$$


The above equation can be written as

$$
\frac{d \ln \delta_{N}(\tau)}{d \tau}\left[\frac{N+1}{2 N}(L-\tau) C_{m r}-c\right]=\frac{N+1}{2 N} C_{m r}
$$

For a given $N$, it can be found from Eq.(15) that $\delta_{N}(\tau)$ is strictly increasing in $\tau$ and it is known that $\left[\frac{N+1}{2 N}(L-\tau) C_{m r}-c\right]>\left[\frac{1}{2}(L-\tau) C_{m r}-c\right]>0$. Thus, the left hand side of Eq. (17) is also strictly increasing in $\tau$. Hence, there exists a finite and unique $\tau^{*}$ which satisfies Eq.(17).

\section{The Optimal PM Policy}

From Theorems 2 to 4, we know there exists unique optimal $N^{*}$ for any given positive $\tau$ and $\eta$ and there exist unique optimal $\tau^{*}$ and $\eta^{*}$ for any given $N$. Thus, we need an algorithm with numerical method to search the optimal solution $\left(N^{*}, \tau^{*}, \eta^{*}\right)$ for the delayed PM model. In the algorithm (presented below), the Nelder-Mead searching method is applied to obtain the optimal solution. In order to verify the proposed model, the decision variables $\eta$ still included in the algorithm even though Theorem 3 shows that $\eta^{*}=1$.

\subsection{The Algorithm For The delayed PM Model}

(1) Let $N=0, \eta_{N}=0, \tau_{N}=0$.

(2) Calculate $C_{\min }=T C\left(N, \tau_{N}, \eta_{N}\right)$ using Eq.(4) or (5).

(3) Let $N=1$, .

(4) Use Nelder-Mead method to Search the values of $\tau_{N}$ over the range of $(0, L]$ and $\eta_{N}$ over the range of $[0,1]$ such that $T C\left(N, \tau_{N}, \eta_{N}\right)$ is minimized;

let $C_{0}=$ minimal value of $T C\left(N, \tau_{N}, \eta_{N}\right)$. 
(5) If $C_{0} \geq C_{\min }$, obtain the optimal solution: $N^{*}=N-1, \tau^{*}=\tau_{N^{*}}, \eta^{*}=\eta_{N^{*}}, T^{*}=\frac{L-\tau^{*}}{N^{*}}$, $T C\left(N^{*}, \tau^{*}, \eta^{*}\right)$, and stop;

else let $N=N+1, C_{\min }=C_{0}$, and go to Step (4).

\section{Numerical Examples}

Numerical examples are performed and the optimal solutions of the delayed PM model are compared to the original PM model developed by Pongpech and Murthy [4]. The system's life is assumed to follow Weibull distribution with scale parameter $\theta=1$ and shape parameter $\beta=2.5$, and 3 while examples of $\beta=1.5$ are illustrated for validation. Let $L=5, C_{m r}=1$ and the coefficients $a, b$, and $c$ of $C_{p m}$ are assigned with different values which satisfy $C_{p m} \geq C_{m r}$ as shown in Table 1.

Table 1 shows the optimal solutions of the examples for the original PM model and the delayed PM model. The following results are obtained for the proposed PM model.

- If $N^{*}>1$, it can be seen that $\tau^{*}>T^{*}$ when $\beta=2.5$ or 3 while $\tau^{*}<T^{*}$ when $\beta=1.5$. The results are reasonable since the hazard rate function has convex shape when $\beta>2$ and it has concave shape when $\beta=1.5$.

- For larger $\beta(=2.5$ or 3$)$, it can be noted that $N^{*}=1$ and $\tau^{*}<T^{*}$ if $c=1.5$ (i.e., large value of $c$ ) and $N^{*}>1$ if $c$ is small $(0.1,0.8)$; for smaller $\beta(=1.5)$, most of the cases have $N^{*}=1$. The following results are the comparison of the delayed model to the original model.

- The delayed PM model has smaller $T C^{*}$ than the original PM model.

- For both models, $\eta^{*}=1$ (in all examples). This result consists with Theorem 3. However, the original model with $\eta^{*}=1$ means that the first PM is a perfect PM (with failure rate reduced to zero, i.e., $\left.\delta^{*}=\lambda(T)\right)$ while the delayed model with $\eta^{*}=1$ means that $\delta_{N}(\tau)=d_{N}(\tau)$ 
- For the original model, the optimal policy is fully-periodic policy (with $N^{*}=1$ ) if $\beta=1.5$ or if $\beta=2.5$ or 3 and $c=1.5$. This result may be caused by the assumption of $C_{p m} \geq C_{m r}$ and the constraint of $\pi<T$ in the original model.

We also analyze the sensitivity of each parameter to the optimal solution of the proposed PM model by using the ANOVA method which is shown in Table 2. It can be found that $\beta$ and $c$ are significantly sensitive to the optimal total maintenance cost $T C$. It can be shown from the results that (1) the hazard rate function do affect the PM policy; (2) the unit cost of failure rate reduced (coefficient $c$ ) in the variable PM cost $\left(C_{p m}\right)$ is more sensitive to the optimal total maintenance cost (TC) than the other coefficients ( $a$ and $b$ ).

\section{Conclusions}

In this paper, the delayed PM model with failure rate reduction is developed for the case of finite time span. The existence of the optimal solution of the delayed PM model is theoretically proved. An algorithm for finding the optimal PM policy is provided. It is shown from the examples that the delayed PM model can provide at least as good optimal solution as the original PM model (while the delayed PM model can result in a better solution in many cases). For Weibull failure distribution with $1<\beta<2$ (hazard rate function with concave shape), the PM program can not be delayed (i.e., $\tau$ can not be greater than $T$ ). The delayed PM model is more suitable than the original model when the system's Weibull failure distribution having large $\beta$ and the PM cost $\left(C_{p m}\right)$ having small value of $c$.

\section{Acknowledgment}

This work was supported in part by National Science Council of Taiwan (Grant No. NSC992221-E-324-006-MY2).

\section{References}


1. Cheng, C.Y., Liu, H.H. and GUO, R. (2008). The periodic preventive maintenance policies with failure rate reduction fora finite time span. Proceedings of the $3^{\text {rd }}$ Asian International Workshop on Advanced Reliability Modeling (AIWARM2008), October 23-25, Taichung, Taiwan, McGraw Hill Int' Enterprises Inc., 362-p369.

2. Cheng, C.Y. and Liu, H.H. (2010). The Finite-Time-Period Preventive Maintenance Policies with Failure Rate Reduction under A Warranty Consideration. Journal of the Chinese Institute of Industrial Engineers, 27(2), 81-89.

3. Jayabalan V. and Chaudhuri, D. (1992). Cost Optimization of Maintenance Scheduling for a System with Assured Reliability. Reliability Engineering and System Safety 41, 21-25.

4. Malik, M.A.K. (1979). Reliable Preventive Maintenance Scheduling. AIIE Transaction, 11, 221-228.

5. Nakagawa, T. (1986). Periodic and Sequential Preventive Maintenance Policies. Journal of Applied Probability, R-23/2, 536-542.

6. Pham, H. and Wang, H. (1996). Invited Review: Imperfect Maintenance. European Journal of Operational Research, 94, 425-438.

7. Pongpech, J. and Murthy, D.N.P. (2006). Optimal Periodic Preventive Maintenance Policy for Leased Equipment. Reliability Engineering \& System Safety, 91, 772-777. 


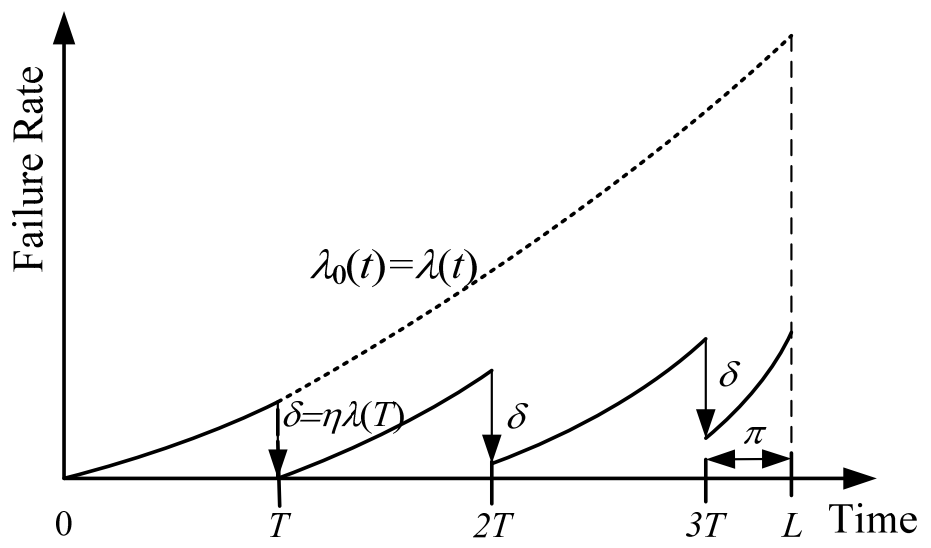

Figure 1. The illustration of the original PM Model with $\mathrm{N}=3$

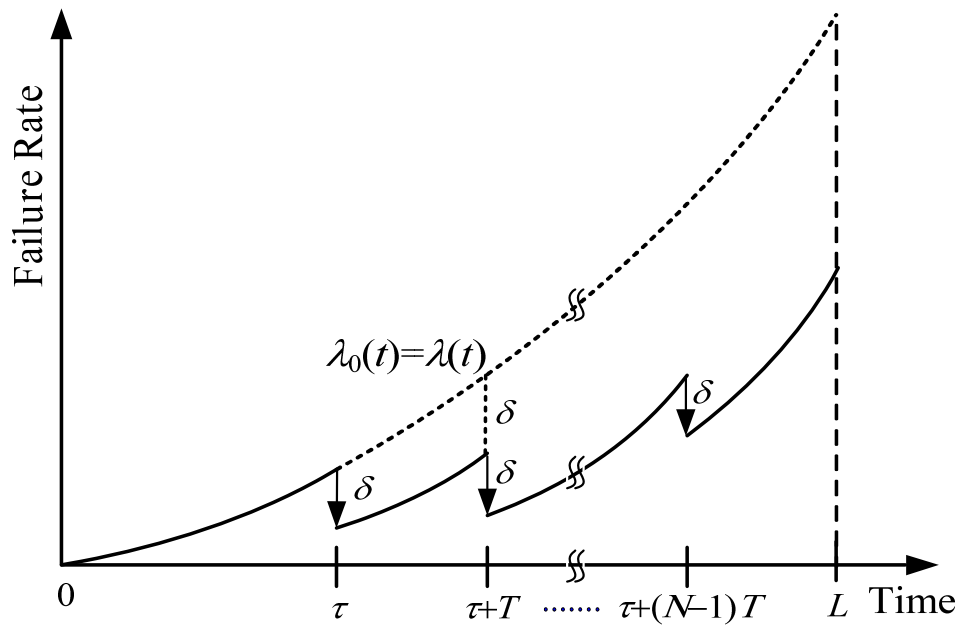

Figure 2. The illustration of the delayed PM Model 
Table 1 . The comparison of the optimal solutions for the delayed model and the original model.

\begin{tabular}{|c|c|c|c|c|c|c|c|c|c|c|c|c|c|c|c|c|c|c|}
\hline \multirow[b]{2}{*}{$a$} & \multirow[b]{2}{*}{$b$} & \multirow[b]{2}{*}{$c$} & \multirow[b]{2}{*}{ Model } & \multicolumn{5}{|c|}{$\beta=1.5$} & \multicolumn{5}{|c|}{$\beta=2.5$} & \multicolumn{5}{|c|}{$\beta=3$} \\
\hline & & & & $N$ & $T$ & $\begin{array}{l}\pi^{*} \\
\tau^{* *}\end{array}$ & $\eta$ & $T C$ & $N$ & $T$ & $\begin{array}{l}\pi^{*} \\
\tau^{* *}\end{array}$ & $\eta$ & $T C$ & $N$ & $T$ & $\begin{array}{c}\pi^{*} \\
\tau^{* *}\end{array}$ & $\eta$ & $T C$ \\
\hline \multirow{2}{*}{1} & \multirow{2}{*}{0.1} & \multirow{2}{*}{0.1} & jed & 2 & 14 & 0.71 & 1 & 58 & 4 & 0.8 & 1.79 & 1 & 1.13 & & 0.48 & 2.13 & 1 & 43.1 \\
\hline & & & Original & 1 & 2.5 & 2.5 & 1 & 6.59 & 2 & 1.96 & 1.08 & 1 & 31.31 & 1 & 3.27 & 1.73 & 1 & 73.81 \\
\hline \multirow{2}{*}{1} & \multirow{2}{*}{0.8} & \multirow{2}{*}{0.1} & jed & 1 & 3.37 & 1.63 & 1 & 6.72 & 3 & 1.03 & 1.91 & 1 & 26.65 & & 0.67 & 2.32 & 1 & 52.56 \\
\hline & & & Original & 1 & 2.5 & 2.5 & 1 & 7.29 & 1 & 2.94 & 2.06 & 1 & 33.00 & 1 & 3.27 & 1.73 & 1 & 74.51 \\
\hline \multirow{2}{*}{1} & \multirow{2}{*}{5} & \multirow{2}{*}{0.1} & Delayed & 1 & 3.37 & 1.63 & 1 & 7.42 & 2 & 1.35 & 2.3 & 1 & 28.84 & & 0.87 & 2.4 & 1 & 58.57 \\
\hline & & & Original & 1 & 2.5 & 2.5 & 1 & 7.99 & 1 & 2.94 & 2.06 & 1 & 33.70 & 1 & 3.27 & 1.73 & 1 & 75.21 \\
\hline \multirow{2}{*}{1} & \multirow{2}{*}{1} & \multirow{2}{*}{0.8} & Delayed & 1 & 3.60 & 1.40 & 1 & 7.31 & 3 & 1.06 & 1.81 & 1 & 35.27 & & 0.53 & 1.83 & 1 & 80.62 \\
\hline & & & Original & 1 & 2.5 & 2.5 & 1 & 8.25 & 2 & 1.68 & 1.64 & 1 & 39.91 & 1 & 2.8 & 2.2 & 1 & 93.17 \\
\hline \multirow{2}{*}{1} & \multirow{2}{*}{.8} & 3 & red & 1 & 3.6 & 1.4 & 1 & 8.01 & 2 & 1.35 & 2.3 & 1 & 38.94 & 3 & 0.92 & 2.23 & 1 & 85.96 \\
\hline & & & Original & 1 & 2.5 & 2.5 & 1 & 8.95 & 1 & 2.52 & 2.48 & 1 & 40.90 & 1 & 2.8 & 2.2 & 1 & 93.87 \\
\hline 1 & & & yed & 1 & 3.6 & 1.4 & 1 & 71 & 2 & 1.35 & 2.3 & 1 & 04 & 5 & 0.92 & 2.23 & 1 & 90.16 \\
\hline & & & Original & 1 & 2.5 & 2.5 & 1 & 9.65 & 1 & 2.52 & 2.48 & 1 & 41.60 & 1 & 2.8 & 2.2 & 1 & 94.57 \\
\hline 1 & & & layed & 1 & 83 & 1.17 & 1 & 8.5 & 1 & 2.9 & 2.1 & 1 & 46.35 & & 2.67 & 2.33 & 1 & 107.04 \\
\hline & & & Original & 1 & 2.5 & 2.5 & 1 & .91 & 1 & 2.5 & 2.5 & 1 & 47.12 & 1 & 2.5 & 2.5 & 1 & 10 \\
\hline 1 & & & Delayed & 1 & 3.83 & 1.17 & 1 & 9.2 & 1 & 2.9 & 2.1 & 1 & 47.05 & & 2.67 & 2.33 & 1 & 107.74 \\
\hline & & & Orig & 1 & 2.5 & 2.5 & 1 & 10.6 & 1 & 2.5 & 2.5 & 1 & 2 & & .5 & 2.5 & 1 & 80 \\
\hline 1 & & & Delayed & 1 & 3.83 & 1.17 & 1 & 9.9 & 1 & 2.9 & 2.1 & 1 & 47.75 & & 2.67 & 2.33 & 1 & 108.44 \\
\hline & & & Oris & 0 & - & - & - & 1.2 & 1 & 2.5 & 2.5 & 1 & 48.52 & & .5 & 2.5 & 1 & 4 \\
\hline & & & Delayed & 1 & 3.37 & 1.63 & 1 & 6.52 & 4 & 0.8 & 1.79 & 1 & 23.13 & & 0.48 & 2.13 & 1 & 46.10 \\
\hline & & & Original & 1 & 2.5 & 2.5 & 1 & 7.09 & 2 & 1.96 & 1.08 & 1 & 32.31 & & 3.27 & 1.73 & 1 & 74.31 \\
\hline & 0.8 & 0 & Delayed & 1 & 3.37 & 1.63 & 1 & 7.22 & 2 & 1.35 & 2.3 & 1 & 27.74 & & 0.67 & 2.31 & 1 & 54.56 \\
\hline & & & Original & 1 & 2.5 & 2.5 & 1 & 7.79 & 1 & 2.94 & 2.06 & 1 & 3.5 & & 3.27 & 1.73 & 1 & 75.01 \\
\hline 15 & 15 & 01 & Del & 1 & .37 & 1.63 & 1 & 7.92 & 2 & 1.35 & 2.3 & 1 & 29.84 & & 0.87 & 2.4 & 1 & 60.07 \\
\hline & & & Original & 1 & 2.5 & 2.5 & 1 & 8.49 & 1 & 2.94 & 2.06 & 1 & 34.2 & & 3.27 & 1.73 & 1 & 75.71 \\
\hline 5 & 0.1 & 0.8 & I & 1 & 3.6 & 1.4 & 1 & 81 & 3 & 1.06 & 1.81 & 1 & 36.77 & 1 & 0.77 & 1.92 & 1 & \begin{tabular}{|l|}
83.16 \\
\end{tabular} \\
\hline & & & Original & 1 & 2.5 & 2.5 & 1 & 8.75 & 1 & 2.52 & 2.52 & 1 & 40.7 & & 2.8 & 2.8 & 1 & 93.67 \\
\hline 15 & 08 & 0 & ed & 1 & 3.6 & 1.4 & 1 & 8.51 & 2 & 1.35 & 2.3 & 1 & 39.94 & $?$ & 0.92 & 2.23 & 1 & 87.46 \\
\hline & & & Original & 1 & 2.5 & 2.5 & 1 & 9.45 & 1 & 2.52 & 2.48 & 1 & 41.4 & & 2.8 & 2.2 & 1 & 94.37 \\
\hline - & 15 & 08 & elayed & 1 & 3.6 & 1.4 & 1 & .21 & 2 & 1.35 & 2.3 & 1 & 42.04 & 5 & 0.92 & 2.23 & 1 & 91.66 \\
\hline & & & Original & 1 & 2.5 & 2.5 & 1 & 10.2 & 1 & 2.52 & 2.48 & 1 & 42.1 & & 2.8 & 2.2 & 1 & 95.07 \\
\hline 1.5 & 0.1 & 1.5 & Delayed & 1 & 3.83 & 1.17 & 1 & 9 & 1 & 2.9 & 2.1 & 1 & 46.85 & 1 & 2.67 & 2.33 & 1 & 107.54 \\
\hline & & & Original & 1 & 2.5 & 2.5 & 1 & 10.4 & 1 & 2.5 & 2.5 & 1 & 47.62 & & 2.5 & 2.5 & 1 & 107.85 \\
\hline 5 & 0.8 & & Delayed & 1 & 3.83 & 1.17 & 1 & 9.7 & 1 & 2.9 & 2.1 & 1 & 7.55 & & 2.67 & 2.33 & 1 & 108.24 \\
\hline & & & Original & 1 & 2.5 & 2.5 & 1 & 11.1 & 1 & 2.5 & 2.5 & 1 & 48.32 & & 2.5 & 2.5 & 1 & 108.55 \\
\hline & .5 & 1 & Delayed & 1 & 3.83 & 1.17 & 1 & 10.4 & 1 & 2.9 & 2.1 & 1 & 48.25 & 1 & 2.67 & 2.33 & 1 & 108.94 \\
\hline & & & Original & 0 & - & - & - & 11.2 & 1 & 2.5 & 2.5 & 1 & 49.02 & & 2.5 & 2.5 & 1 & 109.25 \\
\hline
\end{tabular}

$* \pi$ is a decision variable of the Original PM model; ${ }^{* *} \tau$ is a decision variable of the Delayed PM model. 
Table 2. The sensitivity analysis of the optimal solution for the delayed PM model using ANOVA

\begin{tabular}{cccccc}
\hline \multicolumn{6}{c}{ Response: TC } \\
\hline \multirow{2}{*}{ Source } & $\begin{array}{c}\text { Sum of } \\
\text { Square }\end{array}$ & DF & $\begin{array}{c}\text { Mean } \\
\text { Square }\end{array}$ & F Value & Prob $>$ F \\
\hline Model & 82081.07 & 9 & 9120.12 & 73.12 & $<0.0001$ \\
$\beta$ & 66719.59 & 2 & 33359.80 & 267.47 & $<0.0001$ \\
$a$ & 41.62 & 1 & 41.62 & 0.33 & 0.5650 \\
$b$ & 691.91 & 3 & 230.64 & 1.85 & 0.1443 \\
$c$ & 14627.95 & 3 & 4875.98 & 39.09 & $<0.0001$ \\
Residual & 10726.19 & 86 & 124.72 & & \\
\hline
\end{tabular}

